\title{
Association study to evaluate TFPI gene in CAD in Han Chinese
}

\author{
Ying Zhao ${ }^{1+}$, Yanbo Yu ${ }^{2 \dagger}$, Maowei Shi ${ }^{1}, X_{i}$ Yang $^{3}$, Xueqi $\mathrm{Li}^{4}$, Feng Jiang ${ }^{4}$, Yundai Chen ${ }^{5^{*}}$ (D) and Xiaoli Tian ${ }^{3^{*}}$
}

\begin{abstract}
Background: Tissue factor pathway inhibitor (TFPI) is the main physiological inhibitor of TF-induced blood coagulation process, and may play essential roles in the pathogenesis of major adverse cardiac events. This study was designed to determine whether the variation of TFPI was related with coronary artery disease (CAD) in the Han Chinese populations.

Methods: A total of 1271 patients with coronary atherosclerosis and 1287 normal individuals from northern China were enrolled in the present study. Four tagging single-nucleotide polymorphisms (SNPs) (rs7586970, rs6434222, rs10153820 and rs8176528) from TFPI were selected and genotyped by direct sequencing. And the genotypes of the above SNPs were determined in all these participants.
\end{abstract}

Results: In the populations from Beijing and Harbin, no significant case-control differences in the frequencies of TFPI polymorphism (rs10153820 and rs8176528) were observed between CAD patients and controls. Meanwhile, two SNPs of TFPI (rs7586970 and rs6434222) were found to be associated with CAD in both groups. In stratified analyses based on gender, smoking, hypertension, diabetes mellitus and hyperlipidemia, we further determined that the investigated genetic variations of the TFPI genes seemed to be related with diabetes mellitus in CAD patients.

Conclusions: Genetic variations of the TFPI genes seem to be related with CAD, which likely cooperate with metabolic risk factor (diabetes mellitus) and play critical roles in the pathogenesis of coronary artery disease.

Keywords: Tissue factor pathway inhibitor, Coronary artery disease, Single nucleotide polymorphism, Han Chinese

\section{Background}

Over the past few years, coronary artery disease (CAD) has become a major public health problem and has been associated with increased mortality globally [1]. Evidence shows that atherosclerosis, a chronic inflammatory disease of the arterial vessel wall, is the main cause of CAD $[2,3]$. During the atherosclerotic process, chronic inflammatory responses are often related with the development of thrombus-mediated acute coronary events. Rupture or erosion of atherosclerotic plaques or endothelial cell damage can cause exposure of subendothelial procoagulants such as tissue factor (TF) to circulating blood, followed by the activation of the coagulation process, leading to thrombin formation and subsequent acute coronary occlusion [4].

\footnotetext{
*Correspondence: ydchen301@163.com; tianxiaoli@pku.edu.cn

${ }^{\dagger}$ Equal contributors

${ }^{5}$ Department of Cardiology, Chinese PLA General Hospital, Beijing 100853, China ${ }^{3}$ Department of Human Population Genetics, Institute of Molecular Medicine, Peking University, Beijing 100871, China

Full list of author information is available at the end of the article
}

TF mediated activation of the coagulation cascade is inhibited by its endogenous physiological inhibitor, tissue factor pathway inhibitor (TFPI) [5, 6]. TFPI is constitutively synthesized by the microvascular endothelial cells. Most of the TFPI is bound to the vascular endothelium and only $20-30 \%$ of TFPI is in free forms. TFPI is a circulating, Kunitz-type protease inhibitor, acting as a natural anticoagulant that plays a major role in atherosclerotic plaques [6]. Studies showed that the administration of exogenous TFPI or of the TFPI gene could reduce the restenosis and prevent the immediate thrombus formation after balloon injury to the rabbit aortic neointima [7-9]. Meanwhile, heterozygous TFPI deficiency in atherosclerosis-prone mice exhibited a greater atherosclerotic burden, increased plaque tissue factor activity and decreased time to occlusive thrombosis after photochemical vascular injury [10, 11]. These studies indicate that TFPI attenuates TF activity and acts as a potential modulator of both atherosclerosis and arterial thrombosis. 
Besides simply counteracting the role of TF, experimental data describes certain novel roles for TFPI, such as innate immunity, angiogenesis and lipid metabolism. In a cecal ligation and puncture model of peritonitis, recombinant human TFPI treated mice showed decreased plasma IL-6 levels and subsequently the mortality rate was improved [12]. Exogenous TFPI at higher physiological concentrations inhibits endothelial cell migration and tube formation in vitro, showing effects of inhibiting angiogenesis $[13,14]$. Besides, TFPI could bind to lipoprotein and therefore was called lipoprotein associated coagulation inhibitor (LACI). In a murine model of flow cessation, upregulation of TFPI has been shown to reduce the development of arterial thrombosis and inhibit vascular remodeling associated with flow interruption [15]. On the contrary, TFPI deficiency demonstrated a greater atherosclerotic burden in atherosclerosis-prone Apo E (-/-) mice [11]. Furthermore, association studies demonstrated that TFPI was significantly higher with older age, male gender, increased low-density lipoprotein(LDL), current smoking and diabetes [16, 17].

The mechanism of coronary artery disease is of a complicated nature. Consistent findings indicated a role for TFPI in the pathogenesis of atherosclerosis development, not only counteracting the role of TF but also acting as an anti-inflammatory, anti-angiogenic and lipid-lowering substance. Searching for the genetic variants has been recognized as an essential strategy for the prediction, prevention and individualized treatment of CAD. Hence, in this study, we are determined to explore whether TFPI polymorphisms could influence the risk of CAD in the Han Chinese populations. We selected four tagging SNPs of TFPI (rs7586970, rs6434222, rs10153820 and rs8176528). The frequencies of TFPI were evaluated in Chinese CAD patients from two geographically isolated regions of northern China.

\section{Methods}

\section{Population and the definition of risk factors}

The cases in the present study were hospitalized patients who accepted X-ray coronary angiography for diagnostic purposes from two medical centers located in Beijing and Harbin. The normal controls were selected among hospital employees and blood donors with normal X-ray coronary angiography from the two medical centers. All subjects were Han Chinese coming from northern China. The inclusion and exclusion criteria of CAD and the diagnostic criteria for relevant risk factors were clearly stated in our previous study [18].

This study was registered at the website www.clinical trials.gov (NCT 02961127) and was approved by the clinical ethical committee of the PLA General Hospital and the ethical committee of Harbin Medical University. And all subjects gave written informed consent before participation.

\section{Genotyping of SNPs}

Details on genotyping have previously been described [18]. Human genomic DNA was extracted from EDTAanticoagulated blood sample on the Magna Pure LC Instrument [19]. In view of the hapmap $(\mathrm{CHB}+\mathrm{JPT})$, the four tagging single-nucleotide polymorphisms (SNPs) of TFPI (rs7586970, rs6434222, rs10153820 and rs8176528) were selected. DNA fragments of 120-180 bp containing the above SNPs were selected and amplified by PCR, with the corresponding primers listed in Table 1.

The amplified DNA fragments were subsequently purified by PEG precipitation and subjected to direct sequencing with a BigDye v3.1 kit and running on ABI 3130XL.

\section{Statistical analysis}

Values are expressed as the mean \pm standard deviation or otherwise stated. Univariate analysis of the general characteristics of the population involves the independent Student $t$ test or chi-square test as applicable. Genotype distribution for single SNPs was analyzed for departure from the Hardy-Weinberg equilibrium using the chi-square test. All statistical analyses involved use of SPSS statistical package version 17.0 (SPSS Inc., Chicago, IL, UAS). The significance level was taken to be $p<0.05$.

\section{Results}

\section{Characteristics of the study population}

The clinical characteristics of all the included individuals are shown in Table 2. Two pairs of CAD patients and non-CAD normal controls were recruited among the Han Chinese from the two hospitals in Beijing and Harbin. One pair (Population 1) was collected from northern China while the other (Population 2)

Table 1 The pairs of PCR primers for amplifications of SNPs for TFPI

\begin{tabular}{|c|c|c|c|}
\hline SNP & Gene & Position & primer \\
\hline \multirow[t]{2}{*}{ rs8176528 } & TFPI & intron & $\begin{array}{l}\text { forward: 5'- CAGTTCGTGTAG } \\
\text { GGTTACTCAT -3' }\end{array}$ \\
\hline & & & $\begin{array}{l}\text { reverse:5'- CCAGAGACTTTAT } \\
\text { GAGTGTCT - } 3^{\prime}\end{array}$ \\
\hline \multirow[t]{2}{*}{ rs10153820 } & TFPI & $5^{\prime}$ upstream region & $\begin{array}{l}\text { forward: 5'-CGTTGGAGGTCTC } \\
\text { TCTTAGT-3' }\end{array}$ \\
\hline & & & $\begin{array}{l}\text { reverse:5'- CTGGGCTGAGTAG } \\
\text { CCAAGTT-3' }\end{array}$ \\
\hline \multirow[t]{2}{*}{ rs6434222 } & TFPI & intron & $\begin{array}{l}\text { forward: 5'-GTTTGGTTCAAGA } \\
\text { GAGGAACT-3' }\end{array}$ \\
\hline & & & $\begin{array}{l}\text { reverse:5'- CATGACTCAGCTG } \\
\text { CCAGGACT-3' }\end{array}$ \\
\hline \multirow[t]{2}{*}{ rs7586970 } & TFPI & Serine to Asn & $\begin{array}{l}\text { forward: 5'- GAAGGCGTTCAG } \\
\text { AAAGACTTGGT-3' }\end{array}$ \\
\hline & & & $\begin{array}{l}\text { reverse:5'-CCCTCAGCATTGAC } \\
\text { CACAGT-3' }\end{array}$ \\
\hline
\end{tabular}


Table 2 Characteristics of study populations

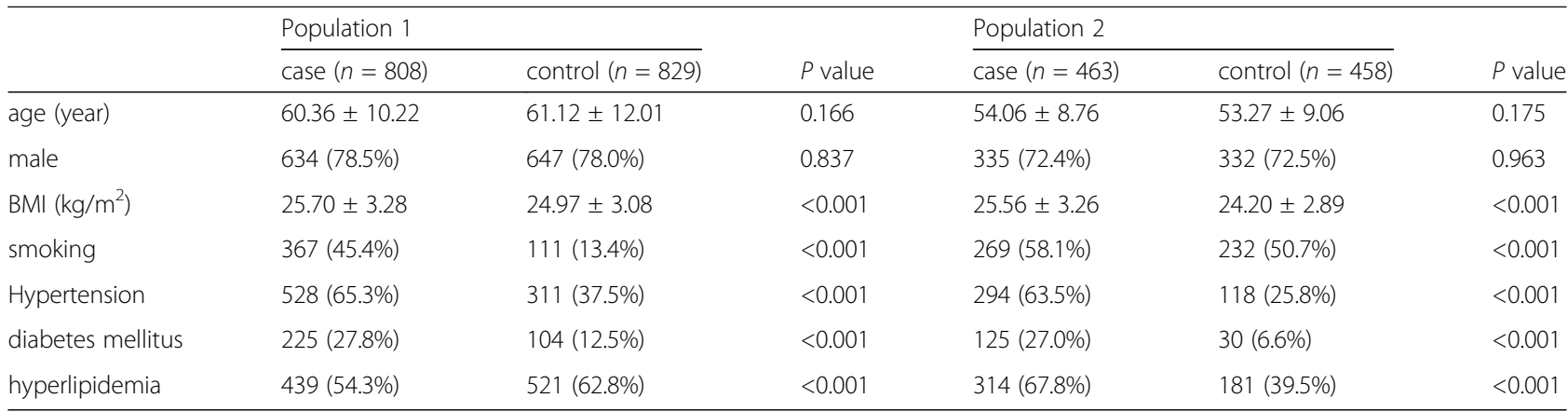

The data were presented as mean \pm SEM (standard error of the mean) for age and BMI as well as No.(percentage) for other factors. $P$ values for age and BMI were calculated from t-test comparing case and control groups within population. $P$ values for gender, smoking, hypertension, diabetes mellitus, hyperlipidemia were calculated from Chi-square test within population. BMl: body mass index, which is calculated by body weight $(\mathrm{Kg}) / \mathrm{height}^{2}\left(\mathrm{~m}^{2}\right)$

was collected from north-eastern China. Population 1 consisted of 808 cases and 829 non-CAD controls whereas Population 2 consisted of 463 cases and 458 non-CAD controls. Both Population 1 and Population 2 were age and gender matched. The risk factors were compared between cases and normal controls by $\mathrm{t}$ test (age and BMI) and Chi-square test (gender, smoking, hypertension, diabetes mellitus and hyperlipidemia).
Genotype distribution and genotype association analysis In both populations from Beijing and Harbin, no significant deviation among the four tagging SNPs of TFPI was found by the Hardy-Weinberg equilibrium test. The distribution of the TFPI genotype among patients and normal controls in both regions is demonstrated in Table 3. No statistically significant differences in the frequencies of rs 8176528 and rs10153820 were obtained between

Table 3 Frequency of TFPI polymorphism in CAD population from two regions

\begin{tabular}{|c|c|c|c|c|c|c|c|}
\hline \multirow[b]{2}{*}{ SNP } & \multirow[b]{2}{*}{ genotype } & \multicolumn{2}{|c|}{ Population 1} & \multirow[b]{2}{*}{$P$} & \multicolumn{2}{|c|}{ Population 2} & \multirow[b]{2}{*}{$P$} \\
\hline & & CAD n (\%) & Non-CAD n (\%) & & CAD n (\%) & Non-CAD n (\%) & \\
\hline \multirow[t]{3}{*}{ rs8176528 } & & 808 & 829 & & 463 & 458 & \\
\hline & GG & $656(81.2)$ & $694(83.7)$ & 0.146 & $380(82.1)$ & $384(83.8)$ & 0.486 \\
\hline & $\begin{array}{l}\mathrm{AA} \\
\mathrm{GA}\end{array}$ & $\begin{array}{l}40(5.0) \\
112(13.8)\end{array}$ & $\begin{array}{l}46(5.6) \\
89(10.7)\end{array}$ & & $\begin{array}{l}18(3.9) \\
65(14.0)\end{array}$ & $\begin{array}{l}21(4.6) \\
53(11.6)\end{array}$ & \\
\hline \multicolumn{2}{|c|}{ Allelic A frequency (\%) } & 11.8 & 10.9 & & 10.9 & 10.3 & \\
\hline \multicolumn{2}{|l|}{ rs10153820 } & 808 & 829 & & 463 & 458 & \\
\hline & GG & $415(51.4)$ & $420(50.7)$ & 0.792 & $285(61.6)$ & $286(62.5)$ & 0.959 \\
\hline & $\begin{array}{l}\mathrm{AA} \\
\mathrm{GA}\end{array}$ & $\begin{array}{l}48(5.9) \\
345(42.7)\end{array}$ & $\begin{array}{l}56(6.7) \\
353(42.6)\end{array}$ & & $\begin{array}{l}81(17.5) \\
97(20.9)\end{array}$ & $\begin{array}{l}79(17.2) \\
93(20.3)\end{array}$ & \\
\hline \multicolumn{2}{|c|}{ Allelic A frequency (\%) } & 27.3 & 28.0 & & 27.9 & 27.4 & \\
\hline \multicolumn{2}{|l|}{ rs6434222 } & 808 & 829 & & 463 & 458 & \\
\hline & $\pi$ & $433(53.6)$ & $496(59.8)$ & $<0.001$ & $235(50.7)$ & $285(62.2)$ & $<0.001$ \\
\hline & $\begin{array}{l}\text { AA } \\
\text { TA }\end{array}$ & $\begin{array}{l}48(5.9) \\
327(40.5)\end{array}$ & $\begin{array}{l}99(11.9) \\
234(28.3)\end{array}$ & & $\begin{array}{l}11(2.4) \\
217(46.9)\end{array}$ & $\begin{array}{l}60(13.1) \\
113(24.7)\end{array}$ & \\
\hline \multicolumn{2}{|c|}{ Allelic A frequency (\%) } & 26.2 & 26.1 & & 25.8 & 25.4 & \\
\hline \multicolumn{2}{|l|}{ rs7586970 } & 808 & 829 & & 463 & 458 & \\
\hline & $\pi$ & $681(84.3)$ & $703(84.8)$ & 0.020 & $384(82.9)$ & $391(85.4)$ & 0.018 \\
\hline & $\begin{array}{l}\text { CC } \\
\text { TC }\end{array}$ & $\begin{array}{l}36(4.5) \\
91(11.2)\end{array}$ & $\begin{array}{l}57(6.9) \\
69(8.3)\end{array}$ & & $\begin{array}{l}21(4.5) \\
58(12.6)\end{array}$ & $\begin{array}{l}32(7.0) \\
35(7.6)\end{array}$ & \\
\hline \multicolumn{2}{|c|}{ Allelic C frequency (\%) } & 10.1 & 11.0 & & 10.8 & 10.8 & \\
\hline
\end{tabular}


CAD cases and non-CAD controls (rs8176528, $p=0.146$ for population 1 and 0.486 for population 2; rs10153820, $p=0.792$ for population 1 and 0.959 for population 2 , Table 3), while statistically significant differences were obtained in the frequencies of rs6434222 and rs7586970 between the two populations from Beijing and Harbin (rs6434222, $p<0.001$ for population 1 and population 2; rs7586970, $p=0.020$ for population 1 and 0.018 for population 2, Table 3).

For better understanding the link between the investigated SNPs and other risk factors in CAD patients, we further performed stratification analyses based on gender, smoking, medical history of hypertension, hyperlipidemia and diabetes mellitus. Due to the influence of diabetes mellitus, a significant difference in the frequencies of TFPI SNPs was obtained in individuals with CAD compared to controls without CAD in our study (shown in Table 4). No obvious differences in the frequencies were obtained for any genotype based on gender (Table 5), smoking (Table 6), hypertension (Table 7), or hyperlipidemia (Table 8 ).

\section{Discussion}

Our present study investigated four tagging SNPs of TFPI in CAD Han Chinese patients from two medical centers in Beijing and Harbin. We demonstrated for the first time that significant differences were drawn in the frequencies of rs7586970 and rs6434222 between CAD

Table 4 Frequencies of TFPI polymorphisms in two populations according to diabetes mellitus

\begin{tabular}{|c|c|c|c|c|c|c|c|c|c|c|c|c|c|}
\hline \multirow[t]{3}{*}{ SNP } & \multirow[t]{3}{*}{ genotype } & \multicolumn{6}{|c|}{ Population 1} & \multicolumn{6}{|c|}{ Population 2} \\
\hline & & \multicolumn{3}{|c|}{ diabetes mellitus } & \multicolumn{3}{|c|}{ Non- diabetes mellitus } & \multicolumn{3}{|c|}{ diabetes mellitus } & \multicolumn{3}{|c|}{ Non- diabetes mellitus } \\
\hline & & $\begin{array}{l}\text { CAD } \\
\mathrm{n}(\%)\end{array}$ & $\begin{array}{l}\text { Non-CAD } \\
\mathrm{n}(\%)\end{array}$ & $P$ & $\begin{array}{l}\text { CAD } \\
\mathrm{n}(\%)\end{array}$ & $\begin{array}{l}\text { Non-CAD } \\
\mathrm{n}(\%)\end{array}$ & $P$ & $\begin{array}{l}\text { CAD } \\
\text { n (\%) }\end{array}$ & $\begin{array}{l}\text { Non-CAD } \\
\mathrm{n}(\%)\end{array}$ & $P$ & $\begin{array}{l}\text { CAD } \\
\text { n (\%) }\end{array}$ & $\begin{array}{l}\text { Non-CAD } \\
\mathrm{n}(\%)\end{array}$ & $P$ \\
\hline \multirow[t]{7}{*}{ Rs8176528 } & GG & 225 & 104 & 0.417 & 583 & 725 & $<0.001$ & 125 & 30 & 0.726 & 338 & 428 & 0.003 \\
\hline & & 199 & 76 & & 457 & 618 & & 65 & 18 & & 315 & 366 & \\
\hline & & $(88.4)$ & $(73.1)$ & & $(78.4)$ & $(85.2)$ & & (52) & $(60.0)$ & & $(93.2)$ & $(85.5)$ & \\
\hline & $\mathrm{AA}$ & 9 & 5 & & 31 & 41 & & 9 & 2 & & 9 & 19 & \\
\hline & & $(4.0)$ & $(4.8)$ & & (5.3) & $(5.7)$ & & $(7.2)$ & (6.7) & & $(2.7)$ & $(4.4)$ & \\
\hline & GA & 17 & 23 & & 95 & 66 & & 51 & 10 & & 14 & 43 & \\
\hline & & $(7.6)$ & $(22.1)$ & & $(16.3)$ & $(9.1)$ & & $(40.8)$ & (33.3) & & $(4.1)$ & $(10.1)$ & \\
\hline \multirow[t]{8}{*}{ Rs10153820 } & GG & 225 & 104 & 0.002 & 583 & 725 & 0.063 & 125 & 30 & 0.038 & 338 & 428 & 0.392 \\
\hline & & 103 & 67 & & 312 & 353 & & 86 & 15 & & 199 & 271 & \\
\hline & & (45.8) & $(64.4)$ & & $(53.5)$ & $(48.7)$ & & (68.8) & (50.0) & & (58.9) & (63.3) & \\
\hline & $A A$ & 27 & 13 & & 21 & 43 & & 19 & 4 & & 62 & 75 & \\
\hline & & (12.0) & $(12.5)$ & & (3.6) & (5.9) & & (15.2) & (13.3) & & (18.3) & (17.5) & \\
\hline & GA & 95 & 24 & & 250 & 329 & & 20 & 11 & & 77 & 82 & \\
\hline & & $(42.2)$ & $(23.1)$ & & $(42.9)$ & $(45.4)$ & & (16.0) & (36.7) & & (22.8) & $(19.2)$ & \\
\hline & & 225 & 104 & & 583 & 725 & & 125 & 30 & & 338 & 428 & \\
\hline \multirow[t]{7}{*}{ Rs6434222 } & $\pi$ & 107 & 53 & 0.803 & 326 & 443 & $<0.001$ & 65 & 17 & 0.675 & 170 & 268 & $<0.001$ \\
\hline & & (47.6) & $(51.0)$ & & $(55.9)$ & $(61.1)$ & & (52.0) & $(56.7)$ & & (50.3) & (62.6) & \\
\hline & $\mathrm{AA}$ & 21 & 8 & & 27 & 91 & & 5 & 2 & & 6 & 58 & \\
\hline & & (9.3) & $(7.7)$ & & $(4.6)$ & (12.6) & & $(4.0)$ & (6.6) & & $(1.8)$ & (13.6) & \\
\hline & TA & 97 & 43 & & 230 & 191 & & 55 & 11 & & 162 & 102 & \\
\hline & & $(43.1)$ & (41.3) & & $(39.5)$ & $(26.3)$ & & (44.0) & (36.7) & & $(47.9)$ & $(23.8)$ & \\
\hline & & 225 & 104 & & 583 & 725 & & 125 & 30 & & 338 & 428 & \\
\hline \multirow[t]{6}{*}{ Rs7586970 } & $\pi$ & 161 & 76 & 0.926 & 520 & 627 & 0.043 & 67 & 13 & 0.065 & 317 & 378 & 0.027 \\
\hline & & (71.6) & (73.1) & & $(89.2)$ & (86.5) & & (53.6) & $(43.3)$ & & (93.8) & $(88.3)$ & \\
\hline & CC & 13 & 5 & & 23 & 52 & & 13 & 8 & & 8 & 24 & \\
\hline & & (5.8) & $(4.8)$ & & (3.9) & $(7.2)$ & & (10.4) & (26.7) & & $(2.4)$ & (5.6) & \\
\hline & $\mathrm{TC}$ & 51 & 23 & & 40 & 46 & & 45 & 9 & & 13 & 26 & \\
\hline & & (22.6) & $(22.1)$ & & (6.9) & (6.3) & & (36.0) & $(30.0)$ & & (3.8) & (6.1) & \\
\hline
\end{tabular}


Table 5 Frequencies of TFPI polymorphisms in two populations according to genders

\begin{tabular}{|c|c|c|c|c|c|c|c|c|c|c|c|c|c|}
\hline \multirow[t]{3}{*}{ SNP } & \multirow[t]{3}{*}{ genotype } & \multicolumn{6}{|c|}{ Population 1} & \multicolumn{6}{|c|}{ Population 2} \\
\hline & & \multicolumn{3}{|l|}{ men } & \multicolumn{3}{|c|}{ women } & \multicolumn{3}{|l|}{ men } & \multicolumn{3}{|c|}{ women } \\
\hline & & $\begin{array}{l}\text { CAD } \\
\text { n (\%) }\end{array}$ & $\begin{array}{l}\text { Non-CAD } \\
\mathrm{n}(\%)\end{array}$ & $P$ & $\begin{array}{l}\text { CAD } \\
\text { n (\%) }\end{array}$ & $\begin{array}{l}\text { Non-CAD } \\
\mathrm{n}(\%)\end{array}$ & $P$ & $\begin{array}{l}\text { CAD } \\
\text { n (\%) }\end{array}$ & $\begin{array}{l}\text { Non-CAD } \\
\text { n (\%) }\end{array}$ & $P$ & $\begin{array}{l}\text { CAD } \\
\text { n (\%) }\end{array}$ & $\begin{array}{l}\text { Non-CAD } \\
\text { n (\%) }\end{array}$ & P \\
\hline \multirow[t]{7}{*}{ Rs8176528 } & GG & 634 & 647 & 0.281 & 174 & 182 & 0.484 & 335 & 332 & 0.327 & 128 & 126 & 0.913 \\
\hline & & 512 & 538 & & 144 & 156 & & 273 & 281 & & 107 & 103 & \\
\hline & & (80.8) & $(83.2)$ & & (82.8) & $(85.7)$ & & (81.5) & (84.7) & & (83.6) & $(81.7)$ & \\
\hline & $\mathrm{AA}$ & 33 & 37 & & 7 & 9 & & 13 & 15 & & 5 & 6 & \\
\hline & & $(5.2)$ & $(5.7)$ & & $(4.0)$ & (5.0) & & (3.9) & $(4.5)$ & & (3.9) & (4.8) & \\
\hline & GA & 89 & 72 & & 23 & 17 & & 49 & 36 & & 16 & 17 & \\
\hline & & $(14.0)$ & $(11.1)$ & & $(13.2)$ & (9.3) & & (14.6) & (10.8) & & $(12.5)$ & (13.5) & \\
\hline \multirow[t]{7}{*}{ Rs10153820 } & GG & 634 & 647 & 0.838 & 174 & 182 & 0.343 & 335 & 332 & 0.988 & 128 & 126 & 0.782 \\
\hline & & 306 & 319 & & 109 & 101 & & 204 & 201 & & 81 & 85 & \\
\hline & & $(48.3)$ & $(49.3)$ & & $(62.6)$ & $(55.5)$ & & $(60.9)$ & $(60.5)$ & & $(63.3)$ & (67.4) & \\
\hline & $\mathrm{AA}$ & 41 & 45 & & 7 & 11 & & 59 & 60 & & 22 & 19 & \\
\hline & & $(6.4)$ & $(7.0)$ & & $(4.0)$ & (6.0) & & (17.6) & (18.1) & & $(17.2)$ & (15.1) & \\
\hline & GA & 287 & 283 & & 58 & 70 & & 72 & 71 & & 25 & 22 & \\
\hline & & (45.3) & $(43.7)$ & & (33.4) & (38.5) & & $(21.5)$ & (21.4) & & (19.5) & $(17.5)$ & \\
\hline \multirow[t]{7}{*}{ Rs6434222 } & $\pi$ & 634 & 647 & $<0.001$ & 174 & 182 & 0.003 & 335 & 332 & $<0.001$ & 128 & 126 & 0.001 \\
\hline & & 312 & 379 & & 121 & 117 & & 166 & 214 & & 69 & 71 & \\
\hline & & $(49.2)$ & (58.6) & & $(69.5)$ & $(64.3)$ & & (49.6) & (64.5) & & (53.9) & $(56.3)$ & \\
\hline & $\mathrm{AA}$ & 41 & 73 & & 7 & 26 & & 7 & 41 & & 4 & 19 & \\
\hline & & (6.5) & $(11.3)$ & & (4.1) & $(14.3)$ & & (2.0) & (12.3) & & (3.1) & (15.1) & \\
\hline & $\mathrm{TA}$ & 281 & 195 & & 46 & 39 & & 162 & 77 & & 55 & 36 & \\
\hline & & $(44.3)$ & $(30.1)$ & & $(26.4)$ & $(21.4)$ & & $(48.4)$ & $(23.2)$ & & $(43.0)$ & (28.6) & \\
\hline \multirow[t]{7}{*}{ Rs7586970 } & $\Pi$ & 634 & 647 & 0.068 & 174 & 182 & 0.263 & 335 & 332 & 0.056 & 128 & 126 & 0.084 \\
\hline & & 537 & 549 & & 144 & 154 & & 293 & 289 & & 91 & 102 & \\
\hline & & (84.7) & $(84.9)$ & & (82.8) & (84.6) & & (87.5) & $(87.0)$ & & (71.1) & $(81.0)$ & \\
\hline & CC & 28 & 44 & & 8 & 13 & & 10 & 21 & & 11 & 11 & \\
\hline & & $(4.4)$ & $(6.8)$ & & $(4.6)$ & $(7.2)$ & & (3.0) & (6.4) & & (8.6) & (8.7) & \\
\hline & $\mathrm{TC}$ & 69 & 54 & & 22 & 15 & & 32 & 22 & & 26 & 13 & \\
\hline & & (10.9) & (8.3) & & (12.6) & $(8.2)$ & & (9.5) & (6.6) & & $(20.3)$ & (10.3) & \\
\hline
\end{tabular}

Calculations were performed with comparison of three different genotypes. Values are the number (percentage) of subjects. After stratification analysis based on gender, no significant association was found between genotype distributions and CAD in CAD patients and non-CAD controls

cases and non-CAD controls from two geographically isolated regions. For better understanding the interaction between genetic variations and other risk factors, stratification analysis was further applied and significant differences in four genotype distributions were found in patients with type 2 diabetes mellitus compared with non-DM controls. These results provided the first evidence that genetic variations of the TFPI genes are associated with the risk of CAD in Han Chinese patients.

The possible interactions between the genetic variations and the onset of CAD have been increasingly studied over the past few years. These studies strongly suggest that genetic variations can contribute to the pathogenesis of $\mathrm{CAD}$, thereby may act as an indicator to predict the onset of the disease. CAD is a chronic inflammatory process resulting from the interactions between lipoprotein metabolism, plaque rupture and thrombosis [20]. Due to the complicated etiology, exploring the possible genetic polymorphisms may be beneficial to understand the variant individual susceptibility to risk factors that cause CAD. In one study, whole genome scans were performed trying to identify the candidate genetic loci related with hypertension, hyperlipidemia, low HDL levels and diabetes [21]. However, up to now, few genetic loci with obvious susceptibility of CAD have been confirmed, emphasizing the diversity and complexity of the disease. 
Table 6 Frequencies of TFPI polymorphisms in two populations according to smoking status

\begin{tabular}{|c|c|c|c|c|c|c|c|c|c|c|c|c|c|}
\hline \multirow[t]{3}{*}{ SNP } & \multirow[t]{3}{*}{ genotype } & \multicolumn{6}{|c|}{ Population 1} & \multicolumn{6}{|c|}{ Population 2} \\
\hline & & \multicolumn{3}{|c|}{ smoking } & \multicolumn{3}{|c|}{ Non- smoking } & \multicolumn{3}{|c|}{ smoking } & \multicolumn{3}{|c|}{ Non- smoking } \\
\hline & & $\begin{array}{l}\text { CAD } \\
n(\%)\end{array}$ & $\begin{array}{l}\text { Non-CAD } \\
\mathrm{n}(\%)\end{array}$ & $P$ & $\begin{array}{l}\text { CAD } \\
\text { n (\%) }\end{array}$ & $\begin{array}{l}\text { Non-CAD } \\
n(\%)\end{array}$ & $P$ & $\begin{array}{l}\text { CAD } \\
\text { n (\%) }\end{array}$ & $\begin{array}{l}\text { Non-CAD } \\
\text { n (\%) }\end{array}$ & $P$ & $\begin{array}{l}\text { CAD } \\
\text { n (\%) }\end{array}$ & $\begin{array}{l}\text { Non-CAD } \\
\mathrm{n}(\%)\end{array}$ & $P$ \\
\hline \multirow[t]{7}{*}{ Rs8176528 } & GG & 367 & 111 & 0.695 & 441 & 718 & 0.273 & 269 & 232 & 0.581 & 194 & 226 & 0.063 \\
\hline & & 298 & 93 & & 358 & 601 & & 233 & 194 & & 147 & 190 & \\
\hline & & $(81.2)$ & $(83.8)$ & & $(81.2)$ & $(83.7)$ & & (86.6) & (83.6) & & (75.8) & $(84.1)$ & \\
\hline & AA & 18 & 6 & & 22 & 40 & & 7 & 9 & & 11 & 12 & \\
\hline & & $(4.9)$ & (5.4) & & (5.0) & (5.6) & & (2.6) & (3.9) & & $(5.7)$ & (5.3) & \\
\hline & GA & 51 & 12 & & 61 & 77 & & 29 & 29 & & 36 & 24 & \\
\hline & & $(13.9)$ & $(10.8)$ & & $(13.8)$ & $(10.7)$ & & $(10.8)$ & (12.5) & & (18.5) & $(10.6)$ & \\
\hline \multirow[t]{7}{*}{ Rs10153820 } & GG & 367 & 111 & 0.998 & 441 & 718 & 0.827 & 269 & 232 & 0.871 & 194 & 226 & 0.988 \\
\hline & & 184 & 56 & & 231 & 364 & & 164 & 146 & & 121 & 140 & \\
\hline & & $(50.1)$ & $(50.5)$ & & $(52.4)$ & $(50.7)$ & & (61.0) & (62.9) & & (62.4) & $(61.9)$ & \\
\hline & AA & 20 & 6 & & 28 & 50 & & 48 & 41 & & 33 & 38 & \\
\hline & & (5.4) & (5.4) & & $(6.3)$ & $(7.0)$ & & $(17.8)$ & $(17.7)$ & & (17.0) & (16.9) & \\
\hline & GA & 163 & 49 & & 182 & 304 & & 57 & 45 & & 40 & 48 & \\
\hline & & $(44.5)$ & $(44.1)$ & & $(41.3)$ & $(42.3)$ & & $(21.2)$ & (19.4) & & (20.6) & $(21.2)$ & \\
\hline \multirow[t]{7}{*}{ Rs6434222 } & $\pi$ & 367 & 111 & 0.021 & 441 & 718 & $<0.001$ & 269 & 232 & $<0.001$ & 194 & 226 & $<0.001$ \\
\hline & & 178 & 64 & & 255 & 432 & & 136 & 132 & & 99 & 153 & \\
\hline & & $(48.5)$ & $(57.7)$ & & $(57.8)$ & $(60.2)$ & & (50.6) & (56.9) & & $(51.0)$ & $(67.7)$ & \\
\hline & $\mathrm{AA}$ & 20 & 11 & & 28 & 88 & & 7 & 39 & & 4 & 21 & \\
\hline & & (5.4) & (9.9) & & (6.3) & $(12.3)$ & & (2.6) & (16.8) & & $(2.1)$ & (9.3) & \\
\hline & TA & 169 & 36 & & 158 & 198 & & 126 & 61 & & 91 & 52 & \\
\hline & & $(46.1)$ & $(32.4)$ & & (35.9) & $(27.5)$ & & $(46.8)$ & (26.3) & & $(46.9)$ & $(23.0)$ & \\
\hline \multirow[t]{7}{*}{ Rs7586970 } & $\pi$ & 367 & 111 & 0.749 & 441 & 718 & 0.064 & 269 & 232 & 0.081 & 194 & 226 & 0.202 \\
\hline & & 306 & 92 & & 375 & 611 & & 221 & 194 & & 163 & 197 & \\
\hline & & $(83.4)$ & (82.9) & & $(85.0)$ & (85.1) & & $(82.2)$ & (83.7) & & $(84.0)$ & $(87.2)$ & \\
\hline & CC & 17 & 7 & & 19 & 50 & & 12 & 18 & & 9 & 14 & \\
\hline & & $(4.6)$ & (6.3) & & $(4.3)$ & $(7.0)$ & & $(4.4)$ & $(7.7)$ & & $(4.6)$ & $(6.2)$ & \\
\hline & $\mathrm{TC}$ & 44 & 12 & & 47 & 57 & & 36 & 20 & & 22 & 15 & \\
\hline & & $(12.0)$ & (10.8) & & $(10.7)$ & (7.9) & & (13.4) & (8.6) & & (11.4) & (6.6) & \\
\hline
\end{tabular}

Calculations were performed with comparison of three different genotypes. Values are the number (percentage) of subjects. After stratification analysis based on smoking status, no significant association was found between genotype distributions and CAD in CAD patients and non-CAD controls

The TFPI gene comprises 9 exons separated by 8 introns with a promoter region. Mature TFPI molecule comprises three tandem Kunitz-type domains. The comprising elements of TFPI are listed as follows: a negatively charged NH2-terminal region connected by the first Kunitz-type domain (K1), a linker domain, a second Kunitz-type domain (K2), a second linker domain, the third Kunitz-type domain (K3) and a positively charged $\mathrm{COOH}$-terminal basic region. As is known, the majority of TFPI is synthesized by vascular endothelial cells and smooth muscle cells [22, 23]. TFPI co-localizes with endothelial cells and macrophages in human atherosclerotic plaques, where it may modulate atherosclerosis and arterial thrombosis by attenuating TF activity [24, 25].
Several investigations focusing on the association between polymorphisms of the TFPI and cardiovascular diseases have been done to make clear the crucial role of TFPI. For instance, in Germany, the polymorphisms of P151L located in TFPI have been put forward in patients with venous thrombosis [26]. Another study carried out in France screened the TFPI gene, V264 M for point sequence variations among patients with acute coronary syndrome. Unfortunately, the result did not demonstrate that the variations of TFPI contribute to acute coronary syndromes [27]. Whether TFPI variations are associated with the susceptibility of CAD still remains unclear.

To explore the link between TFPI gene variations and coronary heart disease, the detection of 4 tagging SNPs 
Table 7 Frequencies of TFPI polymorphisms in two populations according to hypertension

\begin{tabular}{|c|c|c|c|c|c|c|c|c|c|c|c|c|c|}
\hline \multirow[t]{3}{*}{ SNP } & \multirow[t]{3}{*}{ genotype } & \multicolumn{6}{|c|}{ Population 1} & \multicolumn{6}{|c|}{ Population 2} \\
\hline & & \multicolumn{3}{|c|}{ hypertension } & \multicolumn{3}{|c|}{ Non-hypertension } & \multicolumn{3}{|c|}{ hypertension } & \multicolumn{3}{|c|}{ Non-hypertension } \\
\hline & & $\begin{array}{l}\text { CAD } \\
\mathrm{n}(\%) \\
\end{array}$ & $\begin{array}{l}\text { Non-CAD } \\
\mathrm{n}(\%)\end{array}$ & $P$ & $\begin{array}{l}\text { CAD } \\
\mathrm{n}(\%)\end{array}$ & $\begin{array}{l}\text { Non-CAD } \\
\mathrm{n}(\%)\end{array}$ & $P$ & $\begin{array}{l}\text { CAD } \\
\mathrm{n}(\%) \\
\end{array}$ & $\begin{array}{l}\text { Non-CAD } \\
\mathrm{n}(\%)\end{array}$ & $P$ & $\begin{array}{l}\text { CAD } \\
\mathrm{n}(\%)\end{array}$ & $\begin{array}{l}\text { Non-CAD } \\
\mathrm{n}(\%)\end{array}$ & $P$ \\
\hline \multirow[t]{7}{*}{ Rs8176528 } & GG & 528 & 311 & 0.074 & 280 & 518 & 0.403 & 294 & 118 & 0.477 & 169 & 340 & 0.707 \\
\hline & & 428 & 271 & & 228 & 423 & & 238 & 100 & & 142 & 284 & \\
\hline & & $(81.1)$ & $(87.1)$ & & $(81.4)$ & $(81.7)$ & & $(81.0)$ & $(84.7)$ & & $(84.0)$ & $(83.5)$ & \\
\hline & AA & 26 & 10 & & 14 & 36 & & 13 & 6 & & 5 & 15 & \\
\hline & & $(4.9)$ & $(3.2)$ & & $(5.0)$ & (6.9) & & (4.4) & $(5.1)$ & & (3.0) & $(4.4)$ & \\
\hline & GA & 74 & 30 & & 38 & 59 & & 43 & 12 & & 22 & 41 & \\
\hline & & $(14.0)$ & (9.7) & & (13.6) & $(11.4)$ & & $(14.6)$ & $(10.2)$ & & $(13.0)$ & $(12.1)$ & \\
\hline \multirow[t]{7}{*}{ Rs10153820 } & GG & 528 & 311 & 0.327 & 280 & 518 & 0.381 & 294 & 118 & 0.744 & 169 & 340 & 0.797 \\
\hline & & 268 & 174 & & 147 & 246 & & 175 & 75 & & 110 & 211 & \\
\hline & & $(50.8)$ & $(55.9)$ & & $(52.5)$ & $(47.5)$ & & $(59.5)$ & (63.6) & & (65.1) & $(62.1)$ & \\
\hline & $\mathrm{AA}$ & 31 & 18 & & 17 & 38 & & 54 & 19 & & 27 & 60 & \\
\hline & & $(5.8)$ & (5.8) & & (6.1) & (7.3) & & (18.4) & (16.1) & & $(16.0)$ & $(17.6)$ & \\
\hline & GA & 229 & 119 & & 116 & 234 & & 65 & 24 & & 32 & 69 & \\
\hline & & $(43.4)$ & (38.3) & & $(41.4)$ & $(45.2)$ & & $(22.1)$ & $(20.3)$ & & (18.9) & $(20.3)$ & \\
\hline \multirow[t]{7}{*}{ Rs6434222 } & $\pi$ & 528 & 311 & $<0.001$ & 280 & 518 & 0.002 & 294 & 118 & $<0.001$ & 169 & 340 & $<0.001$ \\
\hline & & 274 & 194 & & 159 & 302 & & 146 & 60 & & 89 & 225 & \\
\hline & & $(51.9)$ & $(62.4)$ & & $(56.8)$ & $(58.3)$ & & $(49.7)$ & (50.8) & & (52.7) & $(66.2)$ & \\
\hline & AA & 33 & 37 & & 15 & 62 & & $8(2.7)$ & 21 & & 3 & 39 & \\
\hline & & (6.3) & (11.9) & & (5.4) & $(12.0)$ & & & (17.8) & & $(1.8)$ & $(11.5)$ & \\
\hline & TA & 221 & 80 & & 106 & 154 & & 140 & 37 & & 77 & 76 & \\
\hline & & $(41.8)$ & $(25.7)$ & & (37.8) & $(29.7)$ & & $(47.6)$ & (31.4) & & (45.5) & $(22.3)$ & \\
\hline \multirow[t]{7}{*}{ Rs7586970 } & $\pi$ & 528 & 311 & 0.250 & 280 & 518 & 0.078 & 294 & 118 & 0.312 & 169 & 340 & 0.158 \\
\hline & & 448 & 262 & & 233 & 441 & & 241 & 98 & & 143 & 293 & \\
\hline & & (84.8) & (84.2) & & (83.3) & $(85.2)$ & & (82.0) & (83.1) & & (84.6) & $(86.2)$ & \\
\hline & $C C$ & 23 & 21 & & 13 & 36 & & 14 & 9 & & 7 & 23 & \\
\hline & & $(4.4)$ & (6.8) & & (4.6) & (6.9) & & $(4.8)$ & (7.6) & & $(4.1)$ & (6.8) & \\
\hline & $\mathrm{TC}$ & 57 & 28 & & 34 & 41 & & 39 & 11 & & 19 & 24 & \\
\hline & & (10.8) & (9.0) & & (12.1) & (7.9) & & $(13.2)$ & (9.3) & & (11.3) & (7.0) & \\
\hline
\end{tabular}

Calculations were performed with comparison of three different genotypes. Values are the number (percentage) of subjects. After stratification analysis based on hypertension, no significant association was found between genotype distributions and CAD in CAD patients and non-CAD controls

(rs7586970, rs6434222, rs10153820 and rs8176528) was executed in this study. And we found that frequencies of rs7586970 and rs6434222 showed significant difference in Chinese CAD patients, indicating that the information of the TFPI gene polymorphism was helpful for evaluating the risk of developing coronary heart disease in Han Chinese. Previously, Jia Yu et al. investigated the link between TFPI-2 gene variations and atherosclerosis in the Chinese population, and two SNPs (rs59805398 and rs34489123) and 5 haplotypes were confirmed to be correlated with CAD. Moreover, TFPI-2 gene polymorphisms might not predict the severity of coronary atherosclerosis [28]. Trine B. Opstad et al. demonstrated a significant influence of the TFPI polymorphisms on thrombin generation, which might be an outcome of the reported genotype-induced alterations in the blood TFPI levels, suggesting a modified risk of atherothrombosis in patients holding the TFPI-399 and TFPI-33 polymorphisms [29]. Didier et al. found that the T-287C variations in the 5 ' regulatory region of the TFPI gene were correlated with significant upregulation of the TFPI molecules, suggesting a positive influence of this polymorphism on the TFPI antigen expression. Though the study demonstrated that the $\mathrm{T}-287 \mathrm{C}$ variations were not correlated with an increased incidence of coronary artery disease, the results have not excluded the possibility that other gene variations in the TFPI may influence this incidence [30]. In the present study, the results showed for 
Table 8 Frequencies of TFPI polymorphisms in two populations according to hyperlipidemia

\begin{tabular}{|c|c|c|c|c|c|c|c|c|c|c|c|c|c|}
\hline \multirow[t]{3}{*}{ SNP } & \multirow[t]{3}{*}{ genotype } & \multicolumn{6}{|c|}{ Population 1} & \multicolumn{6}{|c|}{ Population 2} \\
\hline & & \multicolumn{3}{|c|}{ hyperlipidemia } & \multicolumn{3}{|c|}{ Non- hyperlipidemia } & \multicolumn{3}{|c|}{ hyperlipidemia } & \multicolumn{3}{|c|}{ Non- hyperlipidemia } \\
\hline & & $\begin{array}{l}\text { CAD } \\
n(\%)\end{array}$ & $\begin{array}{l}\text { Non-CAD } \\
\mathrm{n}(\%)\end{array}$ & $P$ & $\begin{array}{l}\text { CAD } \\
\mathrm{n}(\%)\end{array}$ & $\begin{array}{l}\text { Non-CAD } \\
n(\%)\end{array}$ & $P$ & $\begin{array}{l}\text { CAD } \\
\text { n (\%) }\end{array}$ & $\begin{array}{l}\text { Non-CAD } \\
\text { n (\%) }\end{array}$ & $P$ & $\begin{array}{l}\text { CAD } \\
n(\%)\end{array}$ & $\begin{array}{l}\text { Non-CAD } \\
\mathrm{n}(\%)\end{array}$ & $P$ \\
\hline \multirow[t]{7}{*}{ Rs8176528 } & GG & 439 & 521 & 0.074 & 369 & 308 & 0.651 & 314 & 181 & 0.580 & 149 & 277 & 0.166 \\
\hline & & 356 & 447 & & 300 & 247 & & 269 & 157 & & 111 & 227 & \\
\hline & & $(81.1)$ & $(85.8)$ & & (81.3) & $(80.2)$ & & $(85.7)$ & $(86.7)$ & & $(74.5)$ & $(81.9)$ & \\
\hline & AA & 21 & 25 & & 19 & 21 & & 10 & 8 & & 8 & 13 & \\
\hline & & $(4.8)$ & $(4.8)$ & & $(5.1)$ & $(6.8)$ & & $(3.2)$ & $(4.4)$ & & $(5.4)$ & $(4.7)$ & \\
\hline & GA & 62 & 49 & & 50 & 40 & & 35 & 16 & & 30 & 37 & \\
\hline & & $(14.1)$ & (9.4) & & (13.6) & $(13.0)$ & & $(11.1)$ & (8.9) & & $(20.1)$ & $(13.4)$ & \\
\hline \multirow[t]{8}{*}{ Rs10153820 } & GG & 439 & 521 & 0.301 & 369 & 308 & 0.848 & 314 & 181 & 0.788 & 149 & 277 & 0.688 \\
\hline & & 217 & 251 & & 198 & 169 & & 196 & 110 & & 89 & 176 & \\
\hline & & $(49.4)$ & $(48.2)$ & & (53.7) & $(54.9)$ & & (62.4) & (60.8) & & $(59.7)$ & $(63.5)$ & \\
\hline & $\mathrm{AA}$ & 20 & 36 & & 28 & 20 & & 53 & 35 & & 28 & 44 & \\
\hline & & $(4.6)$ & (6.9) & & $(7.6)$ & (6.5) & & (16.9) & (19.3) & & (18.8) & (15.9) & \\
\hline & GA & 202 & 234 & & 143 & 119 & & 65 & 36 & & 32 & 57 & \\
\hline & & $(46.0)$ & $(44.9)$ & & (38.7) & (38.6) & & (20.7) & (19.9) & & $(21.5)$ & (20.6) & \\
\hline & & 439 & 521 & & 369 & 308 & & 314 & 181 & & 149 & 277 & \\
\hline \multirow[t]{6}{*}{ Rs6434222 } & $\pi$ & 252 & 321 & 0.002 & 181 & 175 & $<0.001$ & 158 & 125 & $<0.001$ & 77 & 160 & $<0.001$ \\
\hline & & $(57.4)$ & $(61.6)$ & & (49.1) & $(56.8)$ & & (50.3) & $(69.1)$ & & $(51.7)$ & $(57.8)$ & \\
\hline & $\mathrm{AA}$ & 28 & 58 & & 20 & 41 & & 6 & 19 & & 5 & 41 & \\
\hline & & $(6.4)$ & (11.1) & & $(5.4)$ & (13.3) & & $(1.9)$ & (10.5) & & (3.4) & $(14.8)$ & \\
\hline & TA & 159 & 142 & & 168 & 92 & & 150 & 37 & & 67 & 76 & \\
\hline & & $(36.2)$ & $(27.3)$ & & (45.5) & (29.9) & & $(47.8)$ & (20.4) & & $(44.9)$ & $(27.4)$ & \\
\hline \multirow[t]{7}{*}{ Rs7586970 } & $\pi$ & 439 & 521 & 0.117 & 369 & 308 & 0.125 & 314 & 181 & 0.064 & 149 & 277 & 0.073 \\
\hline & & 372 & 449 & & 309 & 254 & & 252 & 160 & & 132 & 231 & \\
\hline & & $(84.7)$ & $(86.2)$ & & (83.7) & $(82.5)$ & & (80.3) & (88.4) & & (88.6) & $(83.4)$ & \\
\hline & CC & 19 & 32 & & 17 & 25 & & 16 & 6 & & 5 & 26 & \\
\hline & & $(4.3)$ & $(6.1)$ & & $(4.6)$ & (8.1) & & (5.1) & (3.3) & & (3.4) & (9.4) & \\
\hline & $\mathrm{TC}$ & 48 & 40 & & 43 & 29 & & 46 & 15 & & 12 & 20 & \\
\hline & & $(11.0)$ & (7.7) & & (11.7) & (9.4) & & (14.6) & (8.3) & & (8.0) & $(7.2)$ & \\
\hline
\end{tabular}

Calculations were performed with comparison of three different genotypes. Values are the number (percentage) of subjects. After stratification analysis based on hyperlipidemia, no significant association was found between genotype distributions and CAD in CAD patients and non-CAD controls

the first time that TFPI gene polymorphism (rs7586970 and rs6434222) could substantially influence the risk of atherosclerosis in Han Chinese.

Most previous studies supported that the higher levels of TFPI is associated with male gender, increased LDL, smoking and diabetes, all of which are widely accepted as cardiovascular risk factors [31]. Hence, we further investigated whether certain selected SNPs in the TFPI gene was related with cardiovascular risk factors (e.g. gender, smoking, medical history of hypertension, diabetes mellitus and hyperlipidemia) among our enrolled participants. And we found that the investigated genetic polymorphisms of the TFPI genes seemed to be related with diabetes mellitus in our enrolled CAD Han Chinese patients.
The association between CAD and diabetes mellitus has been well established. However, the detailed underlying mechanism accounting for this association has not been fully investigated. Evidence showed that patients with diabetes mellitus were related with faster aortic stenosis progression, endothelial dysfunction, higher coronary artery calcium scores and aortic valve calcification $[32,33]$. The accelerated atherosclerotic process presented in patients with type 2 diabetes mellitus might be a consequence of permanent blood hyperglycemia [34]. Chronic blood hyperglycemia may result in the glycosylation of albumin, which has been confirmed to promote the production of TFPI in endothelial cells and monocytes [35]. In chronic hyperglycemia particularly in patients 
with microalbuminuria, the binding of advanced glycated end products could promote the infiltration of peripheral monocytes into the early atherosclerotic lesions and therefore induce an intravascular oxidative stress response, resulting in increased TFPI activity in vitro [36, 37]. Several studies reported that significantly higher TFPI plasma levels have been found in CAD patients complicated with T2DM compared to uncomplicated CAD patients [38-40]. Increased TFPI plasma levels reflect endothelial damage or impaired binding of TFPI to vascular endothelial cells by glycosaminoglycans since TFPI is predominantly produced by vascular endothelium [16, 37]. Thus, the possible expression alterations of TFPI due to genetic polymorphisms, might lead to a hypercoagulable state in CAD patients, which might be more essential for CAD patients complicated with diabetes.

In our study, the possible link between TFPI genetic polymorphism and other metabolic risk factors (e.g. gender, smoking, hypertension and hyperlipidemia) was investigated, and the results showed no evidence indicating a relationship between TFPI variations and those risk factors. However, it should be noteworthy that the participants were volunteers selected from two regions of China, and may not stand for the whole population. Hence, the association of TFPI with cardiovascular risk factors should be analyzed in more ethnic groups and in larger populations in our future studies. In addition, one recent meta-analysis demonstrated that the traditional risk factors associated with culprit plaque rupture (CPR) may vary depending on the clinical presentation of the patients. For example, hypertension was the sole clinical risk factor accounting for the ST-elevated myocardial infarction (STEMI), while advanced age, diabetes mellitus and hyperlipidemia were the candidate clinical predictors in unstable angina and non ST-elevated myocardial infarction (NSTEMI). Whether the association between TFPI polymorphism and risk factors vary depending on different clinical presentations remains unknown [41]. Further investigations are needed to make clear whether TFPI variations are related with certain subtypes of CAD.

\section{Conclusions}

In summary, we identified two new variations located in the TFPI gene among the present population of Han Chinese CAD patients. In addition, genetic polymorphisms of the TFPI gene are likely to be related with type 2 diabetes mellitus in CAD patients. Further investigations are needed to define whether interventions that target TFPI expression and activity by SNPs might retard or reverse the progression of CAD in Han Chinese patients.

\section{Abbreviations}

TFPI: Tissue factor pathway inhibitor; CAD: Coronary artery disease; LACl: Lipoprotein associated coagulation inhibitor; PCl: Percutaneous coronary intervention; CPR: Culprit plaque rupture.

\section{Acknowledgements}

We are very grateful for the helpful contribution from the Department of Human Population Genetics, Institute of Molecular Medicine of Peking University.

\section{Funding}

This study was funded by a grant from National Natural Science Foundation of China (81670486) and the research foundation for young scientist of Jinan Military General Hospital (2016QN03).

\section{Availability of data and materials}

The datasets used and/or analyzed during the current study are available from the corresponding author on reasonable request.

\section{Authors' contributions}

Conception and design of the study: YDC XLT. Data acquisition: YZ FJ XQL $X Y$. Data management and analysis: YZ YBY MWS. Reagents/materials/ analysis tools preparation: XLT XQL FJ. Manuscript drafting/editing: YZ YBY. YZ and YBY contributed equally in this study. All authors read and approved the final manuscript.

\section{Ethics approval and consent to participate}

This study was approved by the clinical ethical committee of the PLA General Hospital and the ethical committee of Harbin Medical University. Written informed consent was obtained from all the participants before enrollment.

\section{Consent for publication}

Not applicable.

\section{Competing interests}

The authors have no financial or other relationship that might lead to a conflict of interest.

\section{Publisher's Note}

Springer Nature remains neutral with regard to jurisdictional claims in published maps and institutional affiliations.

\section{Author details}

'Department of Geriatrics, Jinan Military General Hospital, Jinan 250031, China. ${ }^{2}$ Department of Gastroenterology, Qilu Hospital of Shandong University, Jinan 250012, China. ${ }^{3}$ Department of Human Population Genetics, Institute of Molecular Medicine, Peking University, Beijing 100871, China. ${ }^{4}$ Department of Cardiology, the Fourth Affiliated Hospital of Harbin Medical University, Harbin 150001, China. ${ }^{5}$ Department of Cardiology, Chinese PLA General Hospital, Beijing 100853, China.

Received: 23 January 2017 Accepted: 12 July 2017

Published online: 17 July 2017

\section{References}

1. Blaha MJ, Cainzos-Achirica M, Greenland P, McEvoy JW, Blankstein R, Budoff MJ, Dardari Z, Sibley CT, Burke GL, Kronmal RA, et al. Role of coronary artery calcium score of zero and other negative risk markers for cardiovascular disease: the multi-ethnic study of atherosclerosis (MESA). Circulation. 2016; 133(9):849-58

2. van der Valk FM, Kuijk C, Verweij SL, Stiekema LC, Kaiser Y, Zeerleder S, Nahrendorf M, Voermans C, Stroes ES: Increased haematopoietic activity in patients with atherosclerosis. European heart journal 2016, 38(6):425-432.

3. McAlpine CS, Swirski FK. Circadian influence on metabolism and inflammation in atherosclerosis. Circ Res. 2016;119(1):131-41.

4. Giesen PL, Rauch U, Bohrmann B, Kling D, Roque M, Fallon JT, Badimon JJ, Himber J, Riederer MA, Nemerson Y. Blood-borne tissue factor: another view of thrombosis. Proc Natl Acad Sci U S A. 1999;96(5):2311-5.

5. Girard TJ, Warren LA, Novotny WF, Likert KM, Brown SG, Miletich JP, Broze GJ Jr. Functional significance of the Kunitz-type inhibitory domains of lipoprotein-associated coagulation inhibitor. Nature. 1989;338(6215):518-20. 
6. Bajaj MS, Birktoft JJ, Steer SA, Bajaj SP. Structure and biology of tissue factor pathway inhibitor. Thromb Haemost. 2001;86(4):959-72.

7. Asada Y, Hara S, Tsuneyoshi A, Hatakeyama K, Kisanuki A, Marutsuka K, Sato Y, Kamikubo Y, Sumiyoshi A. Fibrin-rich and platelet-rich thrombus formation on neointima: recombinant tissue factor pathway inhibitor prevents fibrin formation and neointimal development following repeated balloon injury of rabbit aorta. Thromb Haemost. 1998;80(3):506-11.

8. St Pierre J, Yang LY, Tamirisa K, Scherrer D, De Ciechi P, Eisenberg P, Tolunay E, Abendschein D. Tissue factor pathway inhibitor attenuates procoagulant activity and upregulation of tissue factor at the site of balloon-induced arterial injury in pigs. Arterioscler Thromb Vasc Biol. 1999;19(9):2263-8.

9. Yang $L Y$, St Pierre J, Scherrer DE, Lasala JM, Walsh RG, Abendschein DR. Comparison of methods for local delivery of tissue factor pathway inhibitor to balloon-injured arteries in rabbits. Coron Artery Dis. 1999;10(5):327-33.

10. Westrick RJ, Bodary PF, Xu Z, Shen YC, Broze GJ, Eitzman DT. Deficiency of tissue factor pathway inhibitor promotes atherosclerosis and thrombosis in mice. Circulation. 2001;103(25):3044-6.

11. Singh R, Pan S, Mueske CS, Witt TA, Kleppe LS, Peterson TE, Caplice NM, Simari RD. Tissue factor pathway inhibitor deficiency enhances neointimal proliferation and formation in a murine model of vascular remodelling. Thromb Haemost. 2003:89(4):747-51.

12. Opal SM, Palardy JE, Parejo NA, Creasey AA. The activity of tissue factor pathway inhibitor in experimental models of superantigen-induced shock and polymicrobial intra-abdominal sepsis. Crit Care Med. 2001;29(1):13-7.

13. Hembrough TA, Swartz GM, Papathanassiu A, Vlasuk GP, Rote WE, Green SJ, Pribluda VS. Tissue factor/factor VIlla inhibitors block angiogenesis and tumor growth through a nonhemostatic mechanism. Cancer Res. 2003; 63(11):2997-3000.

14. Hembrough TA, Ruiz JF, Papathanassiu AE, Green SJ, Strickland DK. Tissue factor pathway inhibitor inhibits endothelial cell proliferation via association with the very low density lipoprotein receptor. J Biol Chem. 2001;276(15): 12241-8.

15. Pan $\mathrm{S}$, Kleppe $L S$, Witt TA, Mueske CS, Simari RD. The effect of vascular smooth muscle cell-targeted expression of tissue factor pathway inhibitor in a murine model of arterial thrombosis. Thromb Haemost. 2004;92(3):495-502.

16. Morange PE, Renucci JF, Charles MA, Aillaud MF, Giraud F, Grimaux M, Juhan-Vague I. Plasma levels of free and total TFPI, relationship with cardiovascular risk factors and endothelial cell markers. Thromb Haemost. 2001;85(6):999-1003.

17. Mitchell $C T$, Kamineni A, Palmas W, Cushman M. Tissue factor pathway inhibitor, vascular risk factors and subclinical atherosclerosis: the multi-ethnic study of atherosclerosis. Atherosclerosis. 2009;207(1):277-83.

18. Zhao Y, Yu Y, Tian X, Yang X, Li X, Jiang F, Chen Y, Shi M. Association study to evaluate FoxO1 and FoxO3 gene in CHD in Han Chinese. PLoS One. 2014;9(1):e86252

19. Tian $X L$, Wang QK. Generation of transgenic mice for cardiovascular research. Methods in molecular medicine. 2006;129:69-81.

20. Rosenson RS, Brewer HB, Rader DJ. Lipoproteins as biomarkers and therapeutic targets in the setting of acute coronary syndrome. Circ Res. 2014;114(12):1880-9.

21. Krushkal J, Ferrell R, Mockrin SC, Turner ST, Sing CF, Boerwinkle E. Genomewide linkage analyses of systolic blood pressure using highly discordant siblings. Circulation. 1999;99(11):1407-10.

22. Bajaj MS, Kuppuswamy MN, Saito H, Spitzer SG, Bajaj SP. Cultured normal human hepatocytes do not synthesize lipoprotein-associated coagulation inhibitor: evidence that endothelium is the principal site of its synthesis. Proc Natl Acad Sci U S A. 1990;87(22):8869-73.

23. Ameri A, Kuppuswamy MN, Basu S, Bajaj SP. Expression of tissue factor pathway inhibitor by cultured endothelial cells in response to inflammatory mediators. Blood. 1992;79(12):3219-26.

24. Javanmard SH, Shahsavarzadeh T, Saadatnia M. Low levels of tissue factor pathway inhibitor increase the risk of cerebral venous thrombosis. Advanced biomedical research. 2015;4:6.

25. Winckers $\mathrm{K}$, ten Cate $\mathrm{H}$, Hackeng TM. The role of tissue factor pathway inhibitor in atherosclerosis and arterial thrombosis. Blood Rev. 2013;27(3): $119-32$

26. Kleesiek K, Schmidt M, Gotting C, Schwenz B, Lange S, Muller-Berghaus G, Brinkmann T, Prohaska W. The 536C $->$ T transition in the human tissue factor pathway inhibitor (TFPI) gene is statistically associated with a higher risk for venous thrombosis. Thromb Haemost. 1999;82(1):1-5.

27. Moatti D, Seknadji P, Galand C, Poirier O, Fumeron F, Desprez S, Garbarz M, Dhermy D, Arveiler D, Evans A, et al. Polymorphisms of the tissue factor pathway inhibitor (TFPI) gene in patients with acute coronary syndromes and in healthy subjects : impact of the V264M substitution on plasma levels of TFPI. Arterioscler Thromb Vasc Biol. 1999;19(4):862-9.

28. Yu J, Liu RL, Luo XP, Shi HM, Ma D, Pan JJ, Ni HC. Tissue factor pathway inhibitor-2 gene polymorphisms associate with coronary atherosclerosis in Chinese population. Medicine. 2015;94(42):e1675.

29. Opstad TB, Pettersen AA, Bratseth V, Arnesen $H$, Seljeflot I. The influence of tissue factor and tissue factor pathway inhibitor polymorphisms on thrombin generation in stable coronary artery disease. Pathophysiol Haemost Thromb. 2010;37(2-4):98-103.

30. Moatti D, Haidar B, Fumeron F, Gauci L, Boudvillain O, Seknadji P, Olliver V, Aumont MC, de Prost D. A new T-287C polymorphism in the $5^{\prime}$ regulatory region of the tissue factor pathway inhibitor gene. Association study of the T-287C and C-399T polymorphisms with coronary artery disease and plasma TFPI levels. Thromb Haemost. 2000;84(2):244-9.

31. Sakkinen PA, Cushman M, Psaty BM, Kuller LH, Bajaj SP, Sabharwal AK, Boineau R, Macy E, Tracy RP. Correlates of antithrombin, protein C, protein S, and TFPI in a healthy elderly cohort. Thromb Haemost. 1998;80(1):134-9.

32. Boden G, Rao AK. Effects of hyperglycemia and hyperinsulinemia on the tissue factor pathway of blood coagulation. Current diabetes reports. 2007;7(3): 223-7.

33. Gentile NT, Vaidyula VR, Kanamalla U, DeAngelis M, Gaughan J, Rao AK. Factor VIla and tissue factor procoagulant activity in diabetes mellitus after acute ischemic stroke: impact of hyperglycemia. Thromb Haemost. 2007; 98(5):1007-13.

34. Calles-Escandon J, Garcia-Rubi E, Mirza S, Mortensen A. Type 2 diabetes: one disease, multiple cardiovascular risk factors. Coron Artery Dis. 1999:10(1):23-30.

35. Carr ME. Diabetes mellitus: a hypercoagulable state. J Diabetes Complicat. 2001;15(1):44-54.

36. Leurs PB, van Oerle R, Wolffenbuttel BH, Hamulyak K. Increased tissue factor pathway inhibitor (TFPI) and coagulation in patients with insulin-dependent diabetes mellitus. Thromb Haemost. 1997;77(3):472-6.

37. Lindahl AK, Sandset PM, Abildgaard U. The present status of tissue factor pathway inhibitor. Blood coagulation \& fibrinolysis : an international journal in haemostasis and thrombosis. 1992;3(4):439-49.

38. Boden G, Vaidyula VR, Homko C, Cheung P, Rao AK. Circulating tissue factor procoagulant activity and thrombin generation in patients with type 2 diabetes: effects of insulin and glucose. J Clin Endocrinol Metab. 2007:92(11):4352-8.

39. Krupinski J, Turu MM, Font MA, Ahmed N, Sullivan M, Rubio F, Badimon L, Slevin M. Increased tissue factor, MMP-8, and D-dimer expression in diabetic patients with unstable advanced carotid atherosclerosis. Vasc Health Risk Manag. 2007;3(4):405-12.

40. El-Hagracy RS, Kamal GM, Sabry IM, Saad AA, Abou el Ezz NF, Nasr HA. Tissue factor, tissue factor pathway inhibitor and factor VII activity in cardiovascular complicated type 2 diabetes mellitus. Oman medical journal. 2010;25(3):173-8.

41. Iannaccone M, Quadri G, Taha S, D'Ascenzo F, Montefusco A, Omede P, Jang IK, Niccoli G, Souteyrand G, Yundai C, et al. Prevalence and predictors of culprit plaque rupture at OCT in patients with coronary artery disease: a meta-analysis. European heart journal cardiovascular Imaging. 2016;17(10): $1128-37$.

\section{Submit your next manuscript to BioMed Central and we will help you at every step:}

- We accept pre-submission inquiries

- Our selector tool helps you to find the most relevant journal

- We provide round the clock customer support

- Convenient online submission

- Thorough peer review

- Inclusion in PubMed and all major indexing services

- Maximum visibility for your research

Submit your manuscript at www.biomedcentral.com/submit
Biomed Central 